\title{
PLATAFORMA FLEXQUEST: UM RECURSO EDUCACIONAL VOLTADO PARA A CONSERVAÇÃO DOS RECURSOS HÍDRICOS
}

\author{
Victor Hugo Moreira de Lima ${ }^{1}$ \\ Otacílio Antunes Santana ${ }^{2}$ \\ Thais Emanuelle Monteiro dos Santos Souza ${ }^{3}$
}

Resumo: A FlexQuest é uma estratégia que visa a construção de conhecimento mais amplo e flexível, a partir de contextos obtidos diretamente da internet. Neste sentido, a presente pesquisa tem por objetivo produzir uma FlexQuest voltada à conservação dos recursos hídricos com base no princípio da Teoria da Aprendizagem Significativa. A pesquisa foi aplicada na Escola de Referência em Ensino Médio Professora Eurídice Cadaval para trinta e cinco estudantes da $1^{\underline{a}}$ série do Ensino Médio. Os resultados da aplicação da FlexQuest foram obtidos por meio da análise dos mapas conceituais, para categorizar o conhecimento dos estudantes. Pode-se inferir que a FlexQuest proporcionou aos discentes uma eventual ampliação da conscientização ambiental sobre a conservação dos recursos hídricos.

Palavras-chave: FlexQuest; Mapa Conceitual; Educação Ambiental; Água; Web 2.0.

Abstract: FlexQuest is a strategy that aims to build broader and more flexible knowledge, from contexts obtained directly from the internet. In this sense, this research aims to produce a FlexQuest aimed at the conservation of water resources based on the principle of Theory of Meaningful Learning. The research was applied at the High School Reference School Professor Eurídice Cadaval for thirty-five students of the 1st grade of High School. The results of applying FlexQuest were obtained through the analysis of concept maps, to categorize the knowledge of students. It can be inferred that FlexQuest provided students with an eventual expansion of environmental awareness about the conservation of water resources.

Keywords: FlexQuest; Conceitual Map; Environmental Education; Water; Web 2.0.

\footnotetext{
${ }^{1}$ Colégio Agrícola Dom Agostinho Ikas - CODAl. E-mail: victor.hg.ml@hotmail.com.

2 Universidade Federal de Pernambuco. E-mail: otaciliosantana@gmail.com

3 Universidade Federal de Pernambuco. E-mail: thaisemanuelle@hotmail.com
} 


\section{Introdução}

A questão ambiental vem ganhando a atenção dos educadores ao trazer a temática ambiental para os ambientes escolares, seja para cumprir os conteúdos programáticos estabelecidos nos Parâmetros Curriculares Nacionais (PCN) ou pelo reconhecimento da importância do tema. Contudo, verifica-se que muitas vezes o conteúdo relacionado ao meio ambiente é abordado com uma didática pouco motivadora e desconexa com a realidade dos estudantes. Segundo a Base Nacional Comum Curricular (BNCC), o tema "água" deve promover no estudante a reflexão sobre a importância dos recursos hídricos para os seres vivos, envolvendo questões como: formas de aproveitamento da água, o desperdício, o reuso, a qualidade, o tratamento e distribuição e os importantes processos vitais dos quais faz parte (BRASIL, 2017). A água é enfatizada como uma das substâncias mais importantes da natureza e a relação da ação antrópica com a escassez de água em qualidade e quantidade (BRASIL, 2000). Torna-se interessante trabalhar este tema com o auxílio de um recurso didático, pois fortalece o estímulo, a interação e a aprendizagem dos estudantes em sala de aula.

No Brasil, a necessidade de relacionar informações obtidas através de recursos midiáticos aos conteúdos curriculares e contextos de ensino e aprendizagem podem ser identificadas nos documentos de diretrizes oficiais para a educação básica (BRASIL, 2000, 2002, 2006, 2013). A importância dada à descentralização das fontes de informação no ensino, assim como, a preocupação em oferecer diferentes contextos de aprendizagem, resulta na formação de um cidadão capaz de atuar de forma independente e crítica em defesa do meio ambiente.

A atual sociedade está envolta em uma evolução tecnológica. Esta evolução levou a mudanças na vida das pessoas, através do uso da tecnologia (AMICHAI-HAMBURGER et al. 2002; COLL; ILLERA, 2010). A partir desta perspectiva, a escola não pode ignorar sua influência na vida dos indivíduos e, assim, poder oferecer uma formação crítica e ativa, já que o professor não é mais o único a apresentar o saber em sala de aula, as tecnologias dão acesso a um grande número de informações, dentro e fora do âmbito escolar (GRANÉ; WILLEM, 2011).

A utilização da internet, especialmente na Educação Ambiental, tem sido reconhecida pelos professores da área como uma potente ferramenta educacional, atuando como fonte de informação e um meio de interação que pode levar a um maior envolvimento do discente (SILVA et al. 2015). É comum os estudantes usarem recursos online para buscar mais informações, trocar ideias, colaborar e ajudar uns aos outros em tarefas escolares, como já foi dito anteriormente, retirando do professor o papel de única fonte de informação em sala de aula. Essa mudança se deve principalmente à chegada da Web 2.0, ou Websocial, onde o usuário não apenas recebe informações da rede (característica Web 1.0), mas também é responsável pela construção delas. $\mathrm{Na}$ sala de aula, o professor e o discente são responsáveis pela seleção, gestão e 
troca de informações, estabelecendo dinâmicas de colaboração e cooperação (COLL; MONEREO, 2010).

Partindo dos conhecimentos prévios dos estudantes é possível introduzir novos conceitos, como o auxílio da Plataforma FlexQuest, a fim de promover a Educação Ambiental. A Teoria da Aprendizagem Significativa foi escolhida como suporte a FlexQuest por proporcionar a interação entre o novo conhecimento e o já existente (MOREIRA, 2011).

A motivação desta pesquisa surgiu a fim de romper com as ideias que relacionam o uso da internet à cópia indiscriminada de sites de pesquisa e de dispersão de estudantes. Desta forma, esta pesquisa objetiva educar e conscientizar os estudantes da $1^{\underline{a}}$ série do Ensino Médio sobre a conservação dos recursos hídricos com o auxílio da FlexQuest.

\section{Metodologia}

\section{Construção da FlexQuest}

A fim de promover a contextualização, a interdisciplinaridade e aumentar a capacidade crítico-reflexiva dos discentes em relação ao contexto ambiental, esta FlexQuest foi construída na versão 2.0 (segunda geração). A FlexQuest abordou a temática "conservação dos recursos hídricos" e está disponível na homepage WIX ${ }^{\circledR}$, na URL: https://victorhgml.wixsite.com/fqrecursoshidricos. A FlexQuest foi construída com fatos e acontecimentos registrados da internet e foi estruturada com os seguintes elementos: contexto, recursos, questões, processos e transferência.

\section{Validação da FlexQuest}

Por ser considerada um recurso didático, foi necessário comprovar o potencial desta ferramenta como promotora da Educação Ambiental, para isso, necessitou de validação. A validação da ferramenta foi feita por meio da aplicação da FlexQuest no mês de junho de 2019 para trinta e cinco estudantes da $1^{\underline{a}}$ série do Ensino Médio da Escola de Referência em Ensino Médio Professora Eurídice Cadaval, localizada no município de Itapissuma, Estado de Pernambuco. Para a validação foram formadas cinco equipes com sete estudantes que cumpriram as atividades da FlexQuest listadas no Quadro 1. 
Quadro 1: Atividades e processos elencados durante a validação da FlexQuest.

\begin{tabular}{|l|l|l|}
\hline \multicolumn{1}{|c|}{ Minicasos } & \multicolumn{1}{|c|}{ Questões } & \multicolumn{1}{c|}{ Transferência } \\
\hline $\begin{array}{l}\text { Conservação dos } \\
\text { recursos hídricos e a } \\
\text { manutenção da } \\
\text { qualidade da água } \\
\text { para consumo. }\end{array}$ & $\begin{array}{l}\text { Considerando a riqueza dos } \\
\text { recursos hídricos brasileiros, o que } \\
\text { pode ocasionar uma grave crise de } \\
\text { água em nosso país? }\end{array}$ & $\begin{array}{l}\text { Reúnam-se em equipe e } \\
\text { realizem uma entrevista com os } \\
\text { professores e funcionários da } \\
\text { sua escola, com o propósito de } \\
\text { obter informações sobre a } \\
\text { relação destes com a água. Em } \\
\text { seguida, vocês devem expor em } \\
\text { sala de aula o trabalho } \\
\text { realizado. }\end{array}$ \\
$\begin{array}{l}\text { Causa da poluição e e } \\
\text { contaminação das } \\
\text { águas. }\end{array}$ & $\begin{array}{l}\text { A poluição da água aliada ao } \\
\text { desperdício tem gerado vários } \\
\text { problemas para a manutenção desse } \\
\text { bem tão precioso. Como podemos } \\
\text { contribuir para a qualidade e uso } \\
\text { responsável da água? }\end{array}$ & \multicolumn{2}{|l}{ Fonger }
\end{tabular}

Fonte: O autor (2020).

Para atestar o potencial da ferramenta em promover a Educação Ambiental, os mapas conceituais foram utilizados como estratégia. Desta forma, os estudantes foram orientados e mobilizados a construírem um mapa conceitual sobre o tema "conservação dos recursos hídricos", antes e após a aplicação da FlexQuest, a fim de perceber as conexões estabelecidas entre os conceitos presentes na estrutura cognitiva dos mesmos para atestar se houve aprendizado significativo. Essa análise dos dados deliberou a recomendação ou contraindicação da FlexQuest para o público-alvo.

Durante o processo de análise dos dados, os mapas conceituais produzidos pelos estudantes foram analisados em categorias e avaliados em satisfatório ou insatisfatório, conforme o Quadro 2:

Quadro 2: Descrição das categorias de análise dos mapas conceituais.

\begin{tabular}{|c|l|}
\hline Categorias & \multicolumn{1}{|c|}{ Síntese descritiva } \\
\hline $\begin{array}{c}\text { Categoria 1: } \\
\text { Diferenciação } \\
\text { Progressiva }\end{array}$ & $\begin{array}{l}\text { Visou analisar se os conceitos abordados nos mapas conceituais se } \\
\text { tornaram âncora para atribuição de significados a novos } \\
\text { conhecimentos. }\end{array}$ \\
\hline $\begin{array}{c}\text { Categoria 2: } \\
\text { Reconciliação } \\
\text { Integrativa }\end{array}$ & $\begin{array}{l}\text { Buscou analisar se os conceitos apresentaram relações entre ideias, } \\
\text { conceitos já estáveis na estrutura cognitiva. }\end{array}$ \\
\hline $\begin{array}{c}\text { Categoria 3: } \\
\text { Hierarquização dos } \\
\text { conceitos }\end{array}$ & $\begin{array}{l}\text { Visou analisar se os conceitos apresentados nos mapas conceituais } \\
\text { possuem uma hierarquização, ou seja, uma sequência entre os } \\
\text { conceitos na forma de "árvore", em vez de ser alinhado (linear). }\end{array}$ \\
\hline $\begin{array}{c}\text { Categoria 4: } \\
\text { Palavras de Ligação }\end{array}$ & $\begin{array}{l}\text { Buscou analisar se os mapas conceituais apresentam palavras ou } \\
\text { frases que formam um sentido lógico com o conceito ao qual se ligam. }\end{array}$ \\
\hline Categoria 5: Estética & $\begin{array}{l}\text { Propôs diagnosticar quais mapas conceituais apresentam uma fácil } \\
\text { leitura, clareza e elementos estéticos que chamem a atenção do leitor. }\end{array}$ \\
\hline
\end{tabular}

Fonte: Adaptado de Trindade e Hartwig (2012).

Durante o processo de análise dos dados, os grupos de estudantes e os mapas conceituais foram identificados, conforme a seguir: 
- GE1, GE2, GE3, GE4 e GE5: para distinguir os grupos de estudantes;

- MC1i, MC2i, MC3i, MC4i e MC5i: para distinguir os mapas conceituais elaborados pelos diferentes grupos de estudantes antes do uso da FlexQuest;

- MC1f, MC2f, MC3f, MC4f e MC5f: para distinguir os mapas conceituais elaborados pelos diferentes grupos de estudantes após o uso da FlexQuest.

\section{Resultados e discussão}

\section{Análise dos mapas conceituais}

As categorias de avaliação dos mapas conceituais com maior número de resultados insatisfatórios foram as categorias: reconciliação integrativa, palavras de ligação e estética. A avaliação das categorias foram adaptadas de Trindade e Hartwing (2012), a partir da avaliação do próprio mapa conceitual.

A análise dos mapas conceituais permitiu a percepção de que, em relação à primeira categoria (diferenciação progressiva), todos os mapas conceituais apresentados foram satisfatórios, conforme o Quadro 3. De acordo com Moreira (2010), a aprendizagem pode ser facilitada através da diferenciação progressiva e da reconciliação integrativa. O princípio da diferenciação progressiva defende que, em primeiro lugar, os conteúdos mais gerais sejam apresentados inicialmente, e os mais específicos sejam gradualmente introduzidos. Nos mapas conceituais elaborados pelos estudantes, pode-se observar que todos começaram com o conceito mais geral referente ao tema "conservação dos recursos hídricos" e, a partir dele, os conceitos mais específicos. Segundo as concepções de Moreira (2010), quando as ideias mais gerais são apresentadas, elas são progressivamente diferenciadas em termos mais específicos de acordo com o princípio da diferenciação progressiva.

Quadro 3: Identificação e síntese da categoria: diferenciação progressiva.

\begin{tabular}{|l|c|c|}
\hline \multicolumn{2}{|c|}{ Mapas conceituais } \\
\hline \multirow{2}{*}{$\begin{array}{c}\text { Categoria 1: diferenciação } \\
\text { progressiva }\end{array}$} & Satisfatória & MC1i; MC1f; MC2i; MC2f; MC3i; MC3f; \\
\cline { 2 - 3 } & Insatisfatória & \\
\hline $\begin{array}{l}\text { Síntese de análise da categoria 1: observou-se que em relação ao princípio da diferenciação } \\
\text { progressiva, todos apresentaram resultados satisfatórios, ou seja, os conceitos gerais foram } \\
\text { apresentados de forma a servir como âncora para a atribuição de novos conceitos. }\end{array}$ \\
\hline
\end{tabular}

Fonte: $O$ autor (2020).

A categoria reconciliação integrativa foi dividida em duas avaliações: satisfatória e insatisfatória, conforme o Quadro 4. Foram considerados satisfatórios, todos os mapas que estavam conforme o princípio da aprendizagem significativa e insatisfatórios os que não apresentaram o mesmo. 
Quadro 4: Identificação e síntese da categoria: reconciliação integrativa.

\begin{tabular}{|c|c|c|}
\hline \multicolumn{2}{|c|}{ Mapas conceituais } \\
\hline \multirow{2}{*}{$\begin{array}{c}\text { Categoria 2: reconciliação } \\
\text { integrativa }\end{array}$} & Satisfatória & MC1i; MC1f; MC2i; MC2f; MC3i; MC3f; MC4i; \\
\cline { 2 - 3 } & Insatisfatória & MC4f. \\
\hline
\end{tabular}

Síntese de análise da categoria 2: observou-se que em relação ao princípio da reconciliação integrativa oito mapas conceituais apresentaram-se satisfatórios. Os outros dois mapas não evidenciaram semelhanças e diferenças entre os conceitos. Os próprios estudantes tinham consciência de que precisavam fazer reconciliações integrativas, mas não conseguiam provavelmente por ser a primeira vez que estavam elaborando um mapa conceitual; por não conhecerem esse princípio e por não exercitarem diariamente essa atividade de relacionar ideias, conceitos já estabelecidos na estrutura cognitiva.

\section{Fonte: O autor (2020).}

Dos dez mapas conceituais, dois não apresentaram sinais de reconciliação integrativa, nos quais Ausubel (2003) estabelecem que o material a ser apresentado explore a relação entre as noções dos estudantes e os faz mostrar diferenças e semelhanças entre os conceitos apresentados.

No momento da elaboração dos mapas, observou-se que os estudantes tinham noção do que era a reconciliação integrativa, mas ao mesmo tempo não conseguiam fazer essas conexões. Esta dificuldade é provavelmente por algumas razões como: primeira vez que eles estavam desenvolvendo um mapa conceitual; não conhece esse princípio e não exercita diariamente a atividade de relacionar ideias, conceitos já estabelecidos na estrutura cognitiva.

Em relação à terceira categoria: hierarquização dos conceitos, observouse que todos os mapas conceituais apresentaram estruturas com níveis adequados desse princípio, ou seja, foi possível encontrar uma ordenação sucessiva dos conceitos, representados no mínimo por três níveis hierárquicos em forma de "árvore", conforme o Quadro 5. Ausubel (2003) afirma que é mais fácil entender os aspectos diferenciados de um todo previamente aprendido, do que formular o geral a partir das suas partes diferenciadas previamente aprendidas. Além disso, os estudantes deixaram bem claro essa hierarquização dos conceitos principalmente no mapa conceitual final, abordando desde o conceito central até suas especificações.

Quadro 5: Identificação e síntese da categoria: hierarquização dos conceitos.

\begin{tabular}{|c|c|c|}
\hline \multicolumn{3}{|c|}{ Mapas conceituais } \\
\hline $\begin{array}{c}\text { Categoria 3: } \\
\text { hierarquização dos } \\
\text { conceitos }\end{array}$ & Satisfatória & MC1i; MC1f; MC2i; MC2f; MC3i; MC3f; MC4i; MC4f; \\
\cline { 2 - 3 } MC5i; MC5f.
\end{tabular}

Fonte: O autor (2020).

Dos dez mapas conceituais, dois apresentaram-se de forma insatisfatória em relação à quarta categoria: palavras de ligação, conforme o Quadro 6. As palavras de ligação formaram um sentido lógico com o conceito ao qual se 
ligaram. As palavras de ligação mostram as relações existentes entre os conceitos e são representadas pela unidade semântica: conceito - palavra (frase) de ligação - conceito (TRINDADE; HARTWING, 2012).

Quadro 6: Identificação e síntese da categoria: palavras de ligação.

\begin{tabular}{|c|c|c|}
\hline \multicolumn{2}{|c|}{ Mapas conceituais } \\
\hline Categoria 4: & Satisfatória & MC1i; MC1f; MC2i; MC2f; MC3i; MC3f; MC4i; MC4f. \\
\cline { 2 - 3 } palavras de ligação & Insatisfatória & MC5i; MC5f. \\
\hline \multicolumn{2}{|c|}{$\begin{array}{l}\text { Síntese de análise da categoria 4: em relação ao princípio das palavras de ligação observou-se } \\
\text { que oito mapas conceituais elaborados pelos estudantes apresentaram resultados satisfatórios. } \\
\text { Esse princípio é fundamental para a diferenciação entre outros tipos de diagramas conceituais, } \\
\text { como mapa mental, esquema, entre outros. }\end{array}$} \\
\hline
\end{tabular}

Fonte: O autor (2020).

$\mathrm{Na}$ categoria estética, seis mapas conceituais não apresentaram uma estética agradável para uma leitura fácil e elementos que chamem a atenção do leitor, conforme o Quadro 7. Esses mapas apresentaram poucos conceitos subsunçores, poucas ramificações e poucos elementos que chamem a atenção do leitor, como por exemplo as cores, dificultando o entendimento dos conceitos relacionados.

Quadro 7: Identificação e síntese da categoria: estética.

\begin{tabular}{|l|c|c|}
\hline \multicolumn{2}{|c|}{ Mapas conceituais } \\
\hline Categoria 5: estética & Satisfatória & MC1i; MC1f; MC2i; MC2f. \\
\cline { 2 - 3 } & Insatisfatória & MC3i; MC3f; MC4i; MC4f; MC5i; MC5f. \\
\hline $\begin{array}{l}\text { Síntese de análise da categoria 5: a partir de uma análise quanto à estética, observou-se que } \\
\text { em relação a esse princípio, seis mapas conceituais elaborados não foram satisfatórios, pois }\end{array}$ \\
não apresentaram elementos que chamem a atenção do leitor, como por exemplo, diferentes \\
figuras geométricas ou cores para diferenciar os conceitos mais inclusivos dos mais gerais, \\
assim como poucas ramificações o que dificulta o entendimento dos conceitos relacionados.
\end{tabular}

Fonte: $O$ autor (2020).

As categorias dos mapas conceituais serviram para diagnosticar as noções prévias e as possíveis implicações na Educação Ambiental, a partir da inserção de um recurso midiático educacional: FlexQuest. Essa análise foi baseada na Teoria da Aprendizagem Significativa de Ausubel (2003), no qual a relação entre o conhecimento novo com o já existente presente na estrutura cognitiva implica em uma aprendizagem significativa. Essa análise foi feita com cada grupo de estudantes.

A Aprendizagem Siginificava envolve uma interação entre o novo e as noções preexistentes na estrutura cognitiva dos educandos (MOREIRA, 2010). Em outras palavras, foi a partir da análise das noções dos estudantes nos mapas conceituais feitos antes e após a intervenção com a FlexQuest que se diagnosticou indícios de uma aprendizagem significativa e por conseguinte conscientização ambiental.

Trabalhar com o tema "conservação dos recursos hídricos" em sala de aula viabilizou a promoção de reflexões relacionadas ao uso racional deste 
recurso. Considerando que a educação é uma das estratégias para a mitigação dos impactos sobre os recursos hídricos por promover a transformação do indivíduo, tornando-o mais consciente e responsável sobre o uso dos recursos naturais (MAIA et al. 2017).

O mapa conceitual inicial (MC1i) e final (MC1f) elaborados pelo grupo de estudantes 1 (GE1) estão apresentados abaixo para diagnosticar as noções desses discentes e as possíveis ressignificação dos mesmos a respeito do tema "conservação dos recursos hídricos" (Figura 1).
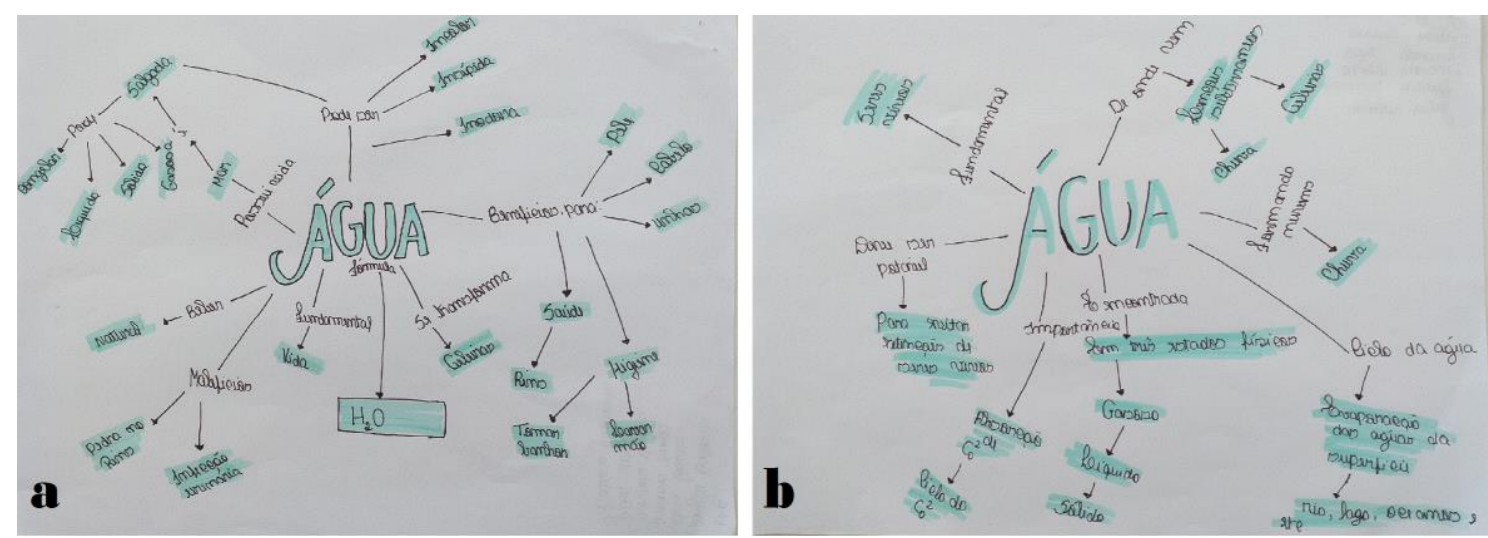

Figura 1: a. Mapa conceitual inicial do grupo de estudantes 1. b. Mapa conceitual final do grupo de estudantes 1.

Tomando uma análise dos conceitos apresentados no MC1i do GE1, observa-se que os estudantes detém apenas conceitos básicos, tais como: estados físicos e a importância da água para a saúde e bem-estar do ser humano. Em relação ao MC1f, elaborado depois da aplicação da FlexQuest, constata-se uma maior amplitude de conceitos relacionados ao tema "conservação dos recursos hídricos", tais como: estrutura química, estados físicos, sua importância para os seres vivos e o ciclo da água. Desta forma, foi possível observar do mapa conceitual sinais aparentes da ampliação cognitiva dos estudantes decorrente da utilização da FlexQuest com a contextualização das teorias na qual foi fundamentada. A partir do produto final elaborado pelos estudantes ficou evidenciado a introdução de novos conceitos.

A mesma análise foi realizada para os demais grupos de estudantes (GE2, GE3, GE4 e GE5), a fim de diagnosticar as possíveis transformações com o suporte midiático apresentado. O mapa conceitual inicial e final elaborado pelo GE2 estão apresentados abaixo (Figura 2). 


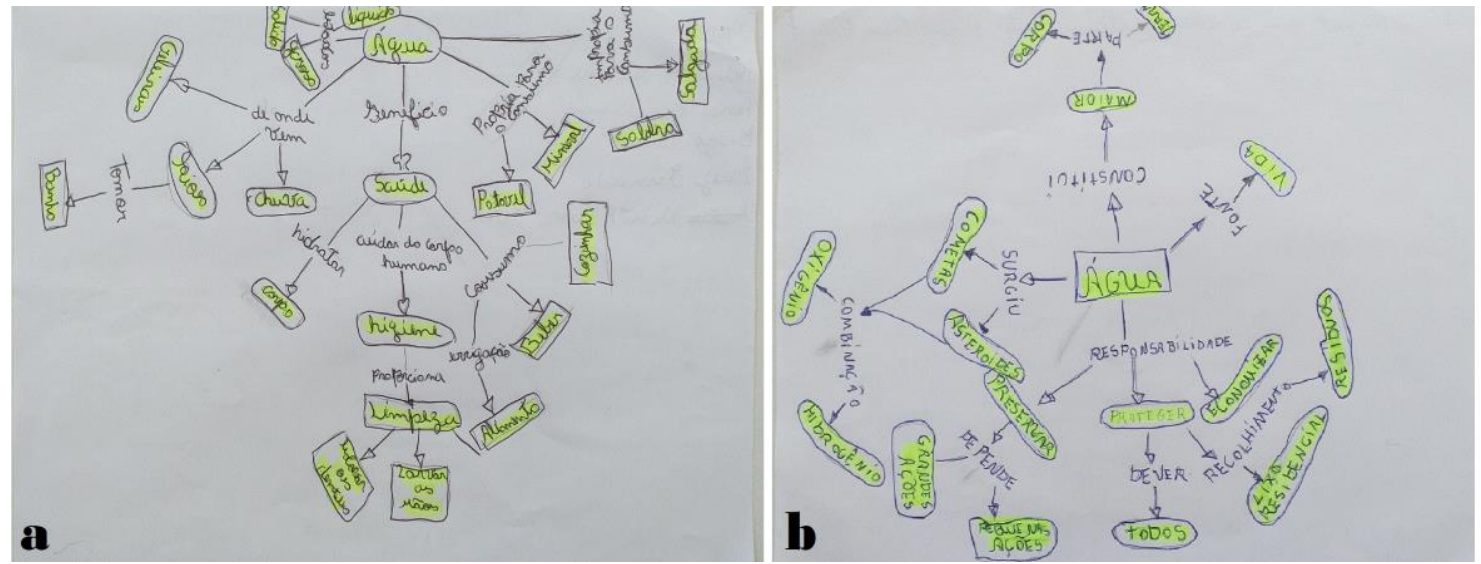

Figura 2: a. Mapa conceitual inicial do grupo de estudantes 2. b. Mapa conceitual final do grupo de estudantes 2 .

Por meio da análise do mapa conceitual inicial (MC2i), percebe-se, novamente que os estudantes apenas esquematizam conceitos básicos referentes aos estados físicos da água e a importância deste recurso para a vida do homem na Terra, demonstrando uma "superficialidade" de conceitos sobre o tema abordado. Por outro lado, o MC2f, elaborado após a aplicação da FlexQuest, apresentou indícios da ampliação de conceitos. Foi possível observar a contextualização que os estudantes fizeram ao abordar os estados físicos da água, a sua origem, a sua importância e a poluição deste recurso.

Em relação ao MC3i, o GE3 descreveu conceitos sem realizar uma relação e diferenciação adequada dos termos mediante os princípios fundamentais de um mapa conceitual. Em relação ao MC3f, observa-se uma maior articulação dos conceitos com o tema, mostrando indícios da ampliação cognitiva dos estudantes após o uso da FlexQuest (Figura 3).

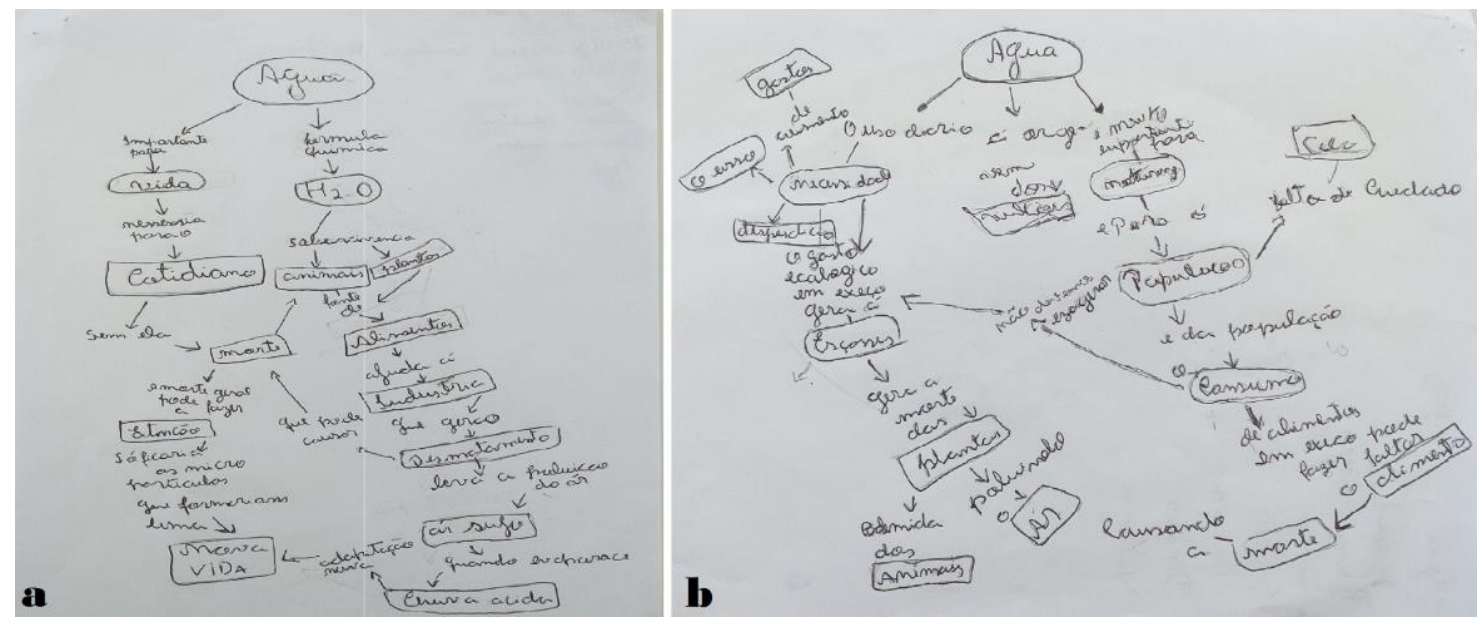

Figura 3: a. Mapa conceitual inicial do grupo de estudantes 3. b. Mapa conceitual final do grupo de estudantes 3 . 
Para o MC4i, ficou evidente uma amplitude de conceitos relacionado ao tema. Contudo, o mapa conceitual apresenta uma deficiência estrutural, comprometendo o princípio da estética. O MC4f também apresentou uma deficiência quanto ao princípio da estética. Contudo, ao ser comparado com o MC4i, percebe-se um nítido enriquecimento de subsunçores envolvendo o conceito central que é a água (Figura 4).

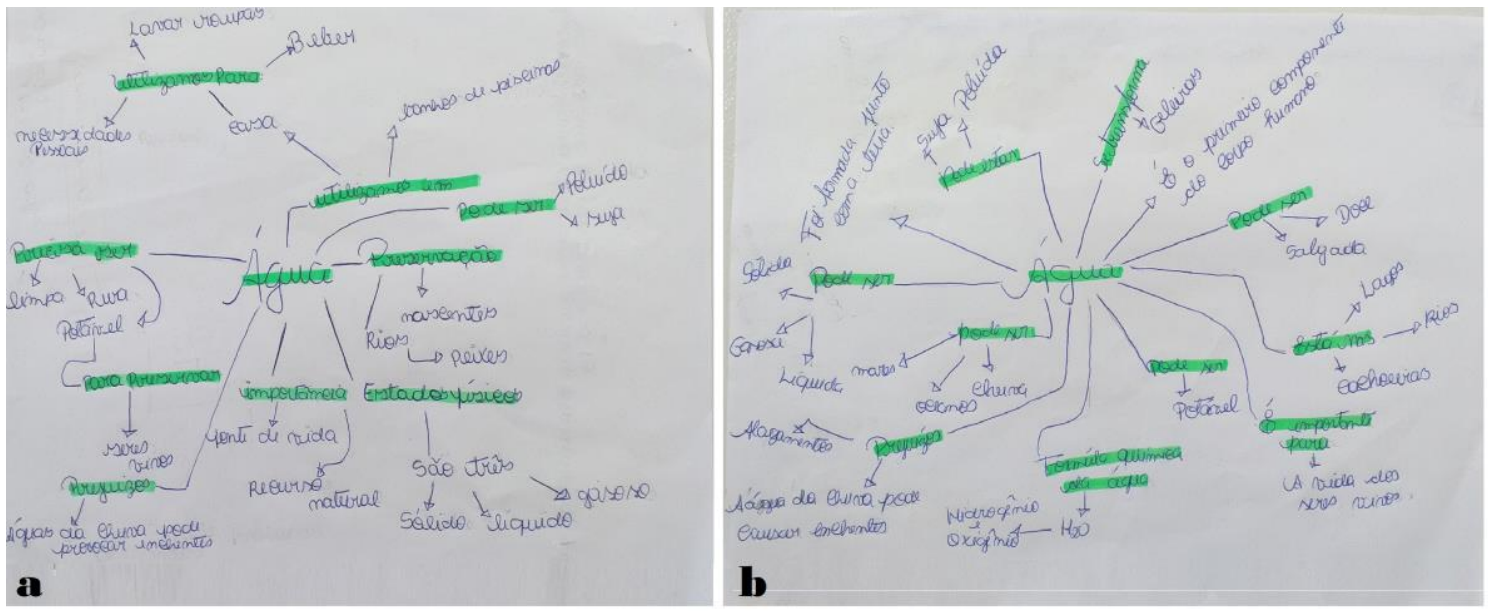

Figura 4: a. Mapa conceitual inicial do grupo de estudantes 4. b. Mapa conceitual final do grupo de estudantes 4 .

Observa-se no MC5i que os estudantes se detém a conceitos dos estados fisícos, falta da água, poluição e a importância da água para a saúde do ser humano. Nesse mesmo mapa também é possível encontrar alguns termos sem conectivos. Em relação ao MC5f elaborado após a aplicação da FlexQuest, ficou evidente uma maior amplitude de conceitos relacionados a temática central, tais como: estrutura química, importância da água para os seres vivos, desperdício, preservação e distribuição da água (Figura 5).
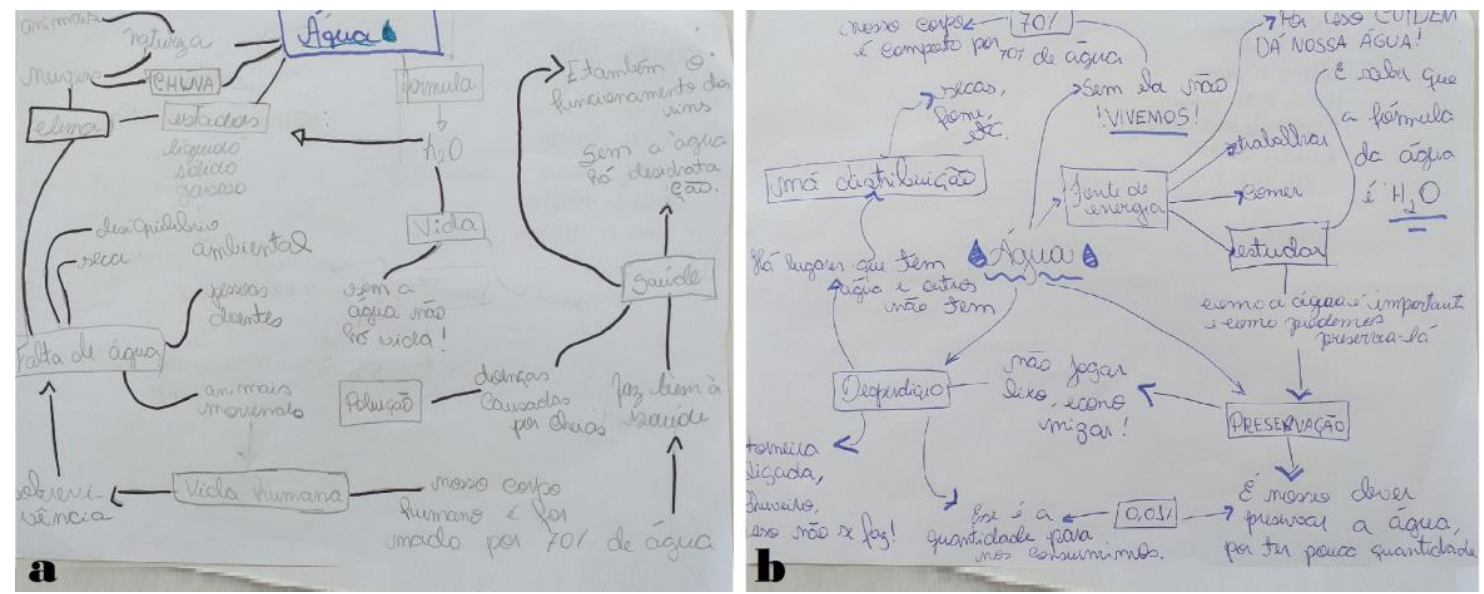

Figura 5: a. Mapa conceitual inicial do grupo de estudantes 5. b. Mapa conceitual final do grupo de estudantes 5 .

Revbea, São Paulo, V. 15, № 5: 47-59, 2020. 
A partir da análise dos mapas conceituais, percebeu-se indícios de uma evolução dos saberes dos estudantes com a utilização do suporte do recurso midiático educacional: FlexQuest. Ao longo do que foi analisado, sustenta-se que as teorizações e interações dos estudantes foram bem melhores após a utilização da FlexQuest, promovendo uma maior reflexão e conscientização sobre os recursos hídricos, ou seja, considera-se que o processo de desenvolvimento da FlexQuest, fez com que os estudantes mobilizassem melhor os seus saberes sobre as questões ambientais.

Indícios mostram que os recursos midiáticos educacionais são vistos como algo essencial para a Educação Ambiental e assim requer dos sujeitos um permanente estado de aprendizagem. Nas análises dos mapas conceituais dos estudantes, é possível observar que os mesmos estão motivados para o uso de tecnologias em sala de aula (RAMOS; AQUINO, 2015; OLIVEIRA et al. 2017). Os recursos midiáticos educacionais são essenciais para o professor modificar sua prática docente e para o estudante se tornar um agente ativo do processo de construção do seu conhecimento (OLIVEIRA et al. 2017; VASCONCELOS; LEÃO, 2016).

Por fim, considerou-se viável a utilização da FlexQuest, contextualizado com a teoria que fundamenta a proposta, para trabalhar o tema "conservação dos recursos hídricos" em sala de aula.

\section{Conclusões}

Para desenvolver um produto educacional (Flex Quest), foi fundamental contextualizar e integrar a teoria da Aprendizagem Significativa e o uso do FlexQuest em sala de aula. Nessa perspectiva, como a pesquisa foi aplicada à estudantes da $1^{1 \underline{a}}$ serie do Ensino Médio, buscou-se identificar as contribuições da FlexQuest para promover a educação e consientização ambiental sobre os recursos hídricos.

Com a articulação da teoria da Aprendizagem Significativa, evidenciaramse indícios de desenvolvimentos metodológicos que permitiram uma aprendizagem significativa. A partir da introdução da FlexQuest como objeto de aprendizagem na Educação Ambiental integrada ao currículo e ao desenvolvimento da prática docente, é possível promover o desdobramento e, consequentemente, a transformação no processo de ensino. Mas para que essa integração e transformação ocorra, é necessário que o professor esteja preparado, pois sabemos que os estudantes estão prontos para usar recurso multimídia.

O material disponibilizado nos casos e minicasos da FlexQuest ocasionou uma boa motivação por parte dos estudantes, bem como uma compreensão inicial sobre as questões ambientais. Nota-se através dos mapas conceituais elaborados pelos discentes que os links disponibilizados na FlexQuest foram efetivamente acessados, bem como contribuíram para a conscientização ambiental. 


\section{Agradecimentos}

Ao gestor, professores, funcionários e estudantes da Escola de Referência em Ensino Médio Professora Eurídice Cadaval que possibilitaram a execução desta pesquisa. Ao Programa de Pós-Graduação em Rede Nacional para Ensino das Ciências Ambientais, Polo Universidade Federal de Pernambuco, por tornar possível o desenvolvimento desta pesquisa.

\section{Referências}

AMICHAI-HAMBURGER, Y.; WAINAPEL, G.; FOX, S. On the Internet no one knows I'm an introvert: extroversion, neuroticism, and Internet interaction. Cyberpsychology \& behavior: the impact of the Internet, multimedia and virtual reality on behavior and society, v. 5, n. 2, p. 125-128, 2002.

AUSUBEL, D.P. Aquisição e retenção de conhecimentos: uma perspectiva cognitiva. Nova Yorque: Plátano, jan. 2003.

BRASIL. Parâmetros Curriculares Nacionais: Ensino Médio. Ministério da Educação (MEC), Secretaria de Educação Média e Tecnológica. Brasília, Ministério da Educação, 2000.

BRASIL. Ministério da Educação. Parâmetros Curriculares Nacionais: Ensino Médio. Brasília: Secretaria da Educação Básica, 2002.

BRASIL. Ministério da Educação. Orientações Curriculares para o Ensino Médio: ciências da natureza, matemática e suas tecnologias. v. 2 Brasília: Secretaria de Educação Básica, 2006.

BRASIL. Diretrizes Curriculares Nacionais da Educação Básica. Brasília: Ministério da Educação (MEC), 2013.

BRASIL. Ministério de Educação e Cultura. Base Nacional Comum Curricular, 2017.

COLL, C.; ILLERA, J.L.R. Alfabetização, novas alfabetizações e alfabetização digital: as TIC no currículo escolar. In: Psicologia da Educação Virtual. Porto Alegre: Artmed, p. 289-310, 2010.

COLL, C.; MONEREO, C. Psicologia da educação virtual: aprender e ensinar com as tecnologias da informação e da comunicação. Porto Alegre: Artmed, p. 6693, 2010.

GRANÉ, M.; WILLEM, C. Aprendendo com a Web 2.0. In: LEÃO, M. B. C. (Ed.). Tecnologias na Educação: Uma Abordagem Crítica para uma Atuação Prática. Recife: UFRPE, p. 87-100, 2011.

MAIA, P. D. S.; OLIVEIRA, R. S.; MARINHO, E. S. M-learning no ensino de ciências: avaliação de objetos educacionais para sensibilização sobre 0 uso racional da água. Redin - Revista Educacional Interdisciplinar, v. 6, n. 1, 2017. 
MOREIRA, M.A. Aprendizaje Significativo Critico. Boletín de Estúdios e Investigació, n. 2 (6) 83-101, 2010.

MOREIRA, M.A. Teorias de aprendizagem. 2. ed. São Paulo: Editora Pedagógica Universitária, 2011.

OLIVEIRA, J.A.B.; SILVA, C.J.; AQUINO, K.A. . Aprendizagem Significativa Crítica e Flexibilidade Cognitiva: diálogo metodológico através da construção e validação de uma ferramenta Flexquest para o ensino de Ecologia na educação básica. Cadernos de Estudos e Pesquisa na Educação Básica, 3, (1), 35-51, 2017.

RAMOS, P.S.; AQUINO, K.A.S. Ações na parte diversificada do currículo: um relato de experiência na perspectiva de uma aprendizagem significativa crítica. Cadernos de Estudos e Pesquisa na Educação Básica, 1, 240-249, 2015.

SILVA, I.G.S.S.; LEÃO, M.B.C.; SOUZA, F.N. Plataforma Flexquest®: Uma estratégia didática para a promoção de flexibilidade cognitiva e interdisciplinaridade com recursos Web 2.0. Revista Ibérica de Sistemas e Tecnologias de Informação, SPE4, 35-49, 2015.

TRINDADE, J.T.; HARTWIG, D.R. Uso combinado de mapas conceituais e estratégias diversificadas de ensino: uma análise inicial das Ligações Químicas. Revista Química Nova na Escola, São Paulo, v. 34, n. 2, p. 83-91, maio, 2012.

VASCONCELOS, F.C.G.C.; LEÃO, M.B.C. Utilização de recursos audiovisuais em uma estratégia Flexquest sobre radioatividade. Investigações em Ensino de Ciências, 1(1), 37-58, 2016. 